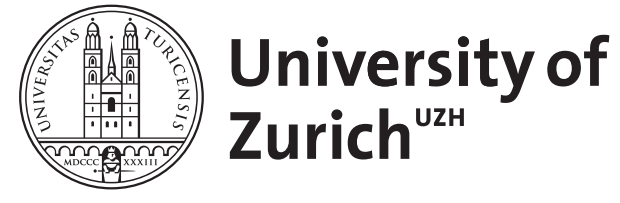

\title{
Zur Konjunktur der analogen Störung im digitalen Bild
}

Flückiger, Barbara

Posted at the Zurich Open Repository and Archive, University of Zurich ZORA URL: https://doi.org/10.5167/uzh-191873

Book Section

Published Version

Originally published at:

Flückiger, Barbara (2004). Zur Konjunktur der analogen Störung im digitalen Bild. In: Schröter, Jens; Böhnke, Alexander. Analog, digital - Opposition oder Kontinuum? : zur Theorie und Geschichte einer Unterscheidung. Bielefeld: 43915, 407-428. 


\section{BARBARA FLÜCKIGER}

\section{ZUR KONJUNKTUR DER ANALOGEN STÖRUNG IM DIGITALEN BILD}

Das digitale Bild hat ein Problem: Es wirkt zu perfekt. Beispielsweise prophezeite Virilio 1993, dass es dereinst „wirklicher erscheinen werde als die Sache, deren Bild es ist." ${ }^{1}$ Zwar ist diese Prophezeiung bis heute nicht eingetreten, dennoch wirft sie ein paar Fragen auf, stößt sie mitten in ein Zentrum derzeitiger Problematiken des digitalen Bildes, vor allem jenes digitalen Bildes, das im Kino erscheint. Wie selbstverständlich verknüpft Virilio zwei Begriffsfelder, die vielgestaltiger nicht sein könnten, ,das (digitale) Bild" und ,die Wirklichkeit'. Und so wird dieser Aufsatz versuchen, diese Beziehung anhand eines Phänomens zu diskutieren, das sich irgendwo zwischen dem digitalen Bild und dem Gegenstand ereignet, auf den es sich bezieht: die Störung.

Noch bevor ein dermaßen waghalsiges Unternehmen in Angriff genommen werden kann, müssen ein paar Begriffe geklärt werden, zumindest die Begriffe, digitales Bild“ und ,Störung'. Von der ,Wirklichkeit' nehme ich erst einmal Abstand und stelle nur die Arbeitshypothese auf, dass sich der Begriff weit gefasst auf eine Beziehung zwischen einer Konstellation in der physikalischen Welt und den Sinnesdaten des menschlichen Wahrnehmungssystems anwenden lässt.

Schiebt sich nun ein technischer Apparat - zum Beispiel eine Filmkamera - zwischen diese physikalische Ausgangsstruktur und unsere Wahrnehmung, ist anzunehmen, dass sich eine irgendwie gestaltete Transformation der Abbildungsbeziehung überlagert, die ich Störung nenne. Obwohl der Begriff, Störung' in der Alltagssprache eine negative Färbung aufweist, werde ich ihn zunächst wertneutral verwenden und im Laufe der Abhandlung seine positiven und negativen Aspekte genauer unter die Lupe nehmen.

Ein transparenter technischer Abbildungsapparat würde keine Transformationen verursachen: das Bild wäre dann identisch mit der wahrgenommenen Ausgangsstruktur, die Störung würde den Wert Null

1 Virilio, Paul: Revolutionen der Geschwindigkeit, Berlin 1993, S. 56. 
annehmen. Am anderen Ende der Skala steht die völlige Transformation bis zur Unkenntlichkeit. Dazwischen sind Myriaden von Abstufungen denkbar. Verschiedene Ursachen können Störungen auslösen, von Elementen des technischen Apparats über die Eigenschaften eines Speichermediums bis $\mathrm{zu}$ den Bearbeitungsprozessen in der Postproduktion. Gespeicherte Informationen können sich durch Alterungsprozesse verändern oder durch die Beschaffenheit eines Wiedergabesystems mit zusätzlichen Störungen angereichert werden.

Das ,digitale Bild' ist ein weites Feld. Es existiert in einer ganzen Palette von Variationen, denen nur wenige Parameter gemein sind: Es besteht aus diskreten Feldern, den Pixeln, deren Eigenschaften durch einen binär kodierten mathematischen Wert definiert sind, und diese Pixel sind gitterförmig - horizontal und vertikal - angeordnet. Eine Reihe von technischen Eigenschaften wie Auflösung, Farbraum oder Kompressionscodes sind für die weitere Betrachtung nicht relevant. Von Bedeutung sind hingegen die Prozesse der Bildentstehung, die ich grob in drei Klassen einteile: 1. digitale Bilddatenakquisition mit einer entsprechenden Kamera; 2. digitale Wandlung eines analogen Bildes durch Scannen; 3. Modellieren eines Bildes in 2D oder 3D auf dem Computer, so genannte CGI (Computer Generated Images). In Compositingverfahren können Visual Effects Artists außerdem verschiedene Bildentstehungsprozesse miteinander kombinieren. ${ }^{2}$ Im Weiteren befassen sich meine Betrachtungen in erster Linie mit jener Art von digitalem Bild, das man fotorealistisch nennt. Damit sind Darstellungsformen gemeint, die sich an den Repräsentationsmodi des fotografischen Bildes orientieren im Unterschied zur klassischen Animation, in der das Gemachte des Bildes ohne Scham ausgestellt wird. Tatsächlich verschwimmen die Grenzen zwischen Fotorealismus und Animation immer mehr, Grenzziehungen zwischen den Darstellungsformen nehmen deshalb einen willkürlichen Charakter an.

In der Praxis kann man nur teilweise aus der Bildanalyse auf die Entstehungsprozesse schließen, im Allgemeinen durch spezifische Störungen, die auf das eine oder andere Verfahren schließen lassen - zum Beispiel den so genannten ,Chattereffekt", bei dem sich verschiedene Bildteile an den Konturen minimal gegeneinander verschieben, durch irreale Farb- oder Lichtsituationen und nicht zuletzt durch Aspekte der Plausibilität, die eine Liveaufnahme am Set als unwahrscheinlich er-

2 Die komplexen Abbildungsverhältnisse, die dabei entstehen, müssen hier ausgeblendet werden. Sie bilden einen Gegenstand meines derzeitigen Forschungsprojekts zu hybriden Formen des filmischen Erzählens. 
scheinen lassen. Wenn Forrest Gump einer Reihe von historisch bedeutsamen Personen begegnet, wenn Protagonisten als Gladiatoren im antiken Rom kämpfen oder sich das Bild des unerreichbaren Geliebten im Blickfeld der Kellnerin Amélie plötzlich verflüssigt, entnehmen einigermaßen mediengewohnte Zuschauer solchen Konstellationen ohne weiteres einen Hinweis auf digitale Bildverfahren. Einige Formen von Störungen jedoch kann nur das geschulte Auge erkennen, indem es das Bild einer genauen Analyse unterwirft. Unplausible Schattenwürfe, das Verhältnis von scharfen und unscharfen Bildteilen, zu betonte oder zu weiche Konturen von abgebildeten Objekten: sie alle und andere mehr können Hinweise auf eine digitale Bildbearbeitung geben.

Schon in diesen wenigen Gedanken zur Störung wird deutlich, dass einige von ihnen, die unmittelbar mit dem Bildgehalt zusammenhängen, einen semantischen Aspekt aufweisen, während andere rein technischer Natur zu sein scheinen und offenbar lediglich die Oberfläche des Bildes verändern. Dazwischen sind wiederum Übergangsphänomene denkbar, die vom einen Spektrum auf das andere überleiten, mithin technische Störungen semantisieren oder umgekehrt. Von diesem Zwischenbereich wird die Rede sein.

In den klassischen Kommunikationsmodellen hatte die Störung unter dem Begriff ,Rauschen' zwar einen technischen Ursprung, entfaltete ihre Relevanz jedoch im semantischen Register, indem sie eine Botschaft veränderte oder eher noch beschädigte, also ausschließlich negativ besetzt war. Im Wirkungsgeschehen des Films jedoch müssen diese modellhaften Komplexitätsreduktionen zurückgewiesen werden. Filmproduktion und -rezeption implizieren schließlich immer eine ästhetische Komponente, die sich sowohl aus Bedeutungsaspekten als auch aus formalen Eigenschaften des dargebotenen Materials speist. Außerdem ist diese ästhetische Komponente sowohl filmhistorisch verortet als auch intermedialen Einflüssen ausgesetzt, erscheint doch das einzelne Filmbild - ob nun analog oder digital - in einem intra- und intertextuellen Bilderverbund.

Im Rahmen meines Forschungsprojekts zu erzählerischen und ästhetischen Einflüssen von digitalen Visual Effects habe ich rund hundert Filme aus der amerikanischen Mainstream-Produktion seit den 80er Jahren bis heute gesichtet, die allesamt diese Visual Effects in ein analoges Umfeld integrieren. Dabei ist eine Reihe von Phänomenen zu Tage getreten, die auf der Nachahmung oder Simulation traditionell analoger Störungen beruhen, Einflüssen des technischen Apparats, die ich im Folgenden darstellen werde. Und zwar geht es bei dieser Darstellung ebenso 
um die ursprünglich physikalisch-technischen Grundlagen wie um die ästhetisch-semantischen Aspekte.

\section{Ein Panoptikum der Phänomene}

\subsection{Korn}

Im Unterschied zum Pixel, der durch eine feste Position innerhalb des geometrischen, horizontalen und vertikalen Bildrasters definiert wird, sind die Körner in der analogen Filmemulsion zufällig verteilt. Sie befinden sich in jedem einzelnen Bild an anderer Stelle und scheinen sich so von Bild zu Bild zu bewegen, zu tanzen. Diese Eigenschaften des Filmkorns bewirken verschiedene Eindrücke, die das Erscheinungsbild des analogen Films charakterisieren. Einerseits führen sie zu mildernden Ausgleichsprozessen, da Konturen oder Bildteile nicht absolut festgelegt sind, sondern sich innerhalb minimaler Toleranzen von Bild zu Bild leicht verschieben. Gleichzeitig reichern sie homogene Flächen mit leichten stochastischen Rastern an und entwickeln damit spezifische Texturen. In dritter Linie markieren sie den Film als Medium und zwar besonders als zeitgebundenes Medium. Indem die Bilder sich durch die Verteilung der Körner minimal voneinander unterscheiden, wirkt auch eine statische Filmeinstellung ohne bewegte Objekte lebendig und scheint eine eigene Form der Zeitlichkeit zu entwickeln. Eine hoch auflösende digitale Aufnahme derselben Situation hingegen mutet auf den ersten Blick tatsächlich transparent wie der Blick aus einem Fenster an. Diese Transparenz - so meinen einige Praktiker, die mit dem Format gearbeitet haben - lasse mit ihrem ungebrochenen Realitätseffekt das Medium nicht geeignet erscheinen, um Geschichten zu erzählen, vielmehr tauge es prinzipiell nur für dokumentarische Zwecke. Die grundsätzlichen Unterschiede sind aber bis heute kaum von praktischer Relevanz, da auch digital produzierte Filme am Ende auf analoges Bildmaterial ausbelichtet und dem Zuschauer in dieser Form präsentiert werden. $^{3}$

Filmkorn wird mit verschiedenen Zielsetzungen auf digitale Bilder gerechnet: 1. um digitales Material an bereits vorhandenes dokumentarisches oder analog produziertes Material anzupassen; 2. um die Histori-

3 Bis heute sind nur wenige Kinofilme ausschließlich digital produziert worden. Wenn solche Filme ab digitalem Master auf DVDs gespielt werden, haben sie tatsächlich nie ein analoges Stadium durchlaufen. 
zität eines digital produzierten Bildes zu behaupten; 3. um aus ästhetischen Gründen einen Filmlook herzustellen.

Fall 1 scheint trivial zu sein. Eines der prominentesten Beispiele für diese Strategie dürfte FORREST GUMP (USA 1994, Robert Zemeckis) sein, in dem der Protagonist in wechselndes dokumentarisches Material eingefügt wird, um historisch bedeutsamen Personen zu begegnen. Hier dienen das gerechnete Korn sowie die Nachahmung anderer filmspezifischer Materialeigenschaften wie Kratzer, eine beschränkte Auflösung und das Kontrastverhalten einer älteren $16 \mathrm{~mm}-\mathrm{s} / \mathrm{w}$-Emulsion dem Zweck, die Figur des Protagonisten unsichtbar in das Bildumfeld zu integrieren. Ein solches Anpassungsprozedere gehört zum Standardrepertoire des Compositing. Auch den computergenerierten Dinosauriern in JURASSIC PARK (USA 1992, Steven Spielberg) wurde ein chaotisch ausgelegtes Kornmuster verpasst, damit sie zum Live-Action-Material passten. Das Verfahren hat wahrnehmungsbedingte Ursachen. Es dient dazu, Differenzen zu minimieren, die sonst die Aufmerksamkeit des Zuschauers auf sich ziehen und Eingriffe in die Integrität des Bildes ins Bewusstsein heben würden. So trivial dieses Verfahren erscheint, so eigenartig ist es bei einiger Reflexion trotzdem. Ist es nicht so, dass die Zuschauer genau wissen, dass Forrest Gump weder Kennedy noch John Lennon getroffen hat? Wissen sie nicht längst bevor sie JURASSIC PARK anschauen, dass einige der Dinosaurier am Computer animiert wurden? Warum muss das digitale Material maskiert werden, wenn gleichzeitig die Computeranimation in den begleitenden Marketingstrategien zum Verkaufsargument erhoben wird?

Fall 2 - Historizität mit filmtypischen Störungen zu behaupten hat bereits eine Geschichte in der traditionellen, analogen Filmproduktion, wie zum Beispiel die ,Wochenschau' in CitIZEN KANE (USA 1941, Orson Welles), die jaulend abbricht, oder ein ,Familienfilm‘ in RAGING BULl (USA 1980, Martin Scorsese), der im ,Super-8-Format einige Jahre im Leben des Protagonisten Jake LaMotta zusammenfasst. Solche Einschübe, die sehr oft medial gestaffelt an einem Bildschirm oder Monitor erscheinen, stützen im Biopic den historisch-öffentlichen Status des Protagonisten. In einem Film wie LE fabUleuX DeSTIN D’AMÉLIE POULAIN (Frankreich 2001, Jean-Pierre Jeunet) hingegen, der massiv mit digitalen Bildtransformationen arbeitet, wird diese Konvention auf ein höheres Abstraktionsniveau gehievt. Dort geht es darum, einen imaginierten öffentlichen Status der tagträumenden Protagonistin in den Film zu implementieren - ein Unterfangen, dessen Absurdität noch gesteigert wird, indem Amélie beim Betrachten ihrer 
eigenen Beerdigung am Fernsehen in Tränen ausbricht. Einen ebenso postmodern überzogenen ,historischen“ Stil setzt Baz Luhrmann in seinem Bohème-Märchen MouLIN Rouge (USA/Australien 2001) ein. In überdrehter Manier überlagert Luhrmann in der Titel- und Eröffnungssequenz ein Maximum an Störungen: grobkörnige Strukturen, Schmutz und Klebestellen, ausgefressene Highlights, diffus auslaufende Konturen bis hin zu Flicker- und Vignettierungseffekten. Die Zeitgestaltung in einer rasenden Fahrt durch das Paris auf der Schwelle zum 20. Jahrhundert ahmt die Schwankungen einer handbetriebenen Stummfilmkamera nach, nicht ohne ihre atemberaubende Beweglichkeit ins AbsurdKörperlose zu transformieren. Schockhaft verzerrt, von expressionistisch bis grotesk wirkt Luhrmanns Stil, den er ,Artificial Reality “ nennt und dessen Künstlichkeit er dem Zuschauer bewusst zumuten will. „He wanted the visual effects to have a handmade feel, to hark back to those olden days rather than be seemless in terms of their digital perfection.“4

MoUlin Rouge passt somit auch in Kategorie 3, Simulation eines Filmlooks aus ästhetischen Gründen. Oft geht es dabei nicht darum, digitales Material unsichtbar an das Vertraute anzupassen, sondern darum, den Filmlook zu überhöhen, ihn auszustellen. Die Filmmaterialien haben sich in punkto Körnigkeit im Lauf der Zeit zu immer unsichtbareren Kornstrukturen hin entwickelt. Die heutigen T-förmigen Körner ( $T$ grains) greifen wie zufällig ineinander und erzeugen so homogen wirkende Farbwolken unterschiedlicher Dichte, sind als Korn im herkömmlichen Sinn also kaum mehr wahrnehmbar. In Opposition zu dieser Entwicklung pushen gegenwärtig Kameraleute Emulsionen bis an ihre Grenzen, unterwerfen sie Stressprogrammen wie dem Bleach-BypassVerfahren, in dem das Bleichbad bei der Farbentwicklung übersprungen wird, um einen körnigen, entsättigten Schmuddellook zu erzeugen - so zum Beispiel für MiNORITY REPORT (USA 2002, Steven Spielberg), einen Film notabene, der eine große Anzahl von computergenerierten Visual Effects einsetzt. Ein ähnliches Verfahren, Color Contrast Enhancement (CCE) genannt, setzte Kameramann Emanuel Lubezki in SLEEPY Hollow (USA 1999, Tim Burton) ein. Im CCE-Prozess wird ebenfalls das Silber in der Emulsion belassen, das Bild wirkt monochrom mit leichten Grün-, Blau- und Brauntönen, betonter Kornstruktur und grafisch wirkenden s/w-Kontrasten, einzig die Farbe Rot - assoziiert mit der Farbe des Blutes - beherrscht in Gewaltszenen fast hyperreal gesättigt das Bild und nimmt damit eine symbolische Bedeutung an.

4 Catherine Martin zitiert nach Fordham, Joe: „Paris by Numbers“, in: Cinefex, Jg. 12, Nr. 86 (2001) S. 16. 
Es scheint paradox, den Compositingtechnikern die Arbeit zu erschweren, indem man die Differenz zwischen kornfreien digitalen Bildteilen und analog gedrehtem Material mit solchen Verfahren maximiert. Schon in den 60er und 70er Jahren des 20. Jahrhunderts gab es in der künstlerisch ambitionierten Fotografie und in gewissen Strömungen des Independent Films eine Tendenz, die Kornstruktur hervorzuheben, teilweise mit dem Ziel grafischer Abstraktion, vor allem aber, um eine höhere Form des Realismus zu erreichen, indem sich dieser Look an der Fotoreportage und am Dokumentarfilm orientierte - mithin also an Produkten, die unter technisch ungünstigen Bedingungen mit wenig Licht und unter großem zeitlichen Druck entstehen. Seither ist diese Ästhetik nie wieder verschwunden, hat sich aber in Werbung und Videoclip zum stylish-modischen Attribut hin entwickelt. In SLEEPY HOLLOW jedoch ist ein solcher Einfluss nicht $\mathrm{zu}$ spüren. Dort orientiert sich die Bildgestaltung vielmehr an einem piktorialen, expressionistischen Stil, steht das unbunte Bild eher für eine düstere Stimmung in einer heil wirkenden, ländlichen Idylle. Für Janusz Kaminski, den Kameramann von MinORITY REPORT, aber ist das Korn die Essenz des Films schlechthin: „To me, movies are a social event [...]. They don't necessarily help us escape realitity, but they offer us a reality that is different from what we see at home. If you can't see or sense grain in the image, you're not experiencing the magic of movies." ${ }^{\text {"5 }}$

\subsection{Motion Blur}

Nicht nur die Körnigkeit, auch die verwischte, unscharfe Aufzeichnung von bewegten Objekten ist eine typische Störung traditioneller Filmaufnahmen. Diese Bewegungsunschärfe - im Fachjargon Motion Blur genannt - ist die Folge einer eigentlich zu geringen Aufzeichnungsrate von 24 Bildern pro Sekunde; zu gering ist diese Rate in Hinblick auf die zeitliche Auflösungsfähigkeit des Auges. Wie mehrheitlich bekannt sein dürfte, wird diese Störung bei der Wiedergabe durch eine Flügelblende so kompensiert, dass der Projektor jedes Bild zweimal auf die Leinwand wirft, damit den Flickereffekt unterdrückt und Bewegungen dennoch fließend erscheinen lässt. Die Filmtechnik hat sich aus ökonomischen Gründen auf diese Aufzeichnungsrate geeinigt, weil man damit Material sparen konnte, sogar sehr viel Material. Mit den derzeitigen digitalen

5 Janusz Kaminski zitiert nach Holben, Jay: „Criminal Intent“, in: American Cinematographer, Jg. 82, Nr. 7 (2002) S. 35. 
Formaten ist diese Sparübung jedoch obsolet geworden, da die Materialkosten im Verhältnis zum Gesamtbudget nicht mehr ins Gewicht fallen.

Während Jahrzehnten sind klassische Visual Effects ohne Motion Blur entstanden. Mit der damals üblichen Technik der Stop-MotionAnimation, bei der die Animatoren Modelle einzelbildweise bewegen und im Ruhezustand aufnehmen, entfällt diese Form der Unschärfe. Es stellt sich ein ganz eigener Bildeindruck ein, den viele mit der spezifischen, ohnehin künstlichen Qualität von mythischen Fabelwesen und magischen Monstern verbinden, wie KING Kong (USA 1933, Ernest B. Schoedsack, Merian C. Cooper) oder die kämpfenden Skelette aus JASON AND the ARgONAUTS (USA 1963, Don Chaffey). Solche Assoziationen sind unerwünscht, wenn die animierten Modelle lebensecht erscheinen sollen. Deshalb haben findige Tüftler seit den 70er Jahren eine Reihe von analogen Methoden ausgedacht, um Motion Blur künstlich zu erzeugen, von der Diffusion bewegter Bildteile durch partielle Fettschichten auf dem Objektiv hin zu Mehrfachbelichtungen. Eine überzeugende und praktikable Lösung hat sich jedoch erst mit dem Computer entwickelt. Kameras konnten nun via Motion Control präzise Bewegungen mehrfach wiederholen, Modelle konnte man mit der Go-Motion-Animation so steuern, dass sie genau während der Aufnahme winzige Bewegungen ausführten. Heute, im Zeitalter der computergenerierten Monster, haben sich Motion-Blur-Tools etabliert, die den Effekt auf die Computeranimation rechnen und den Eindruck in Abhängigkeit von Objekt- und Kamerabewegung sowie Belichtungszeit perfekt nachbilden.

Eigentlich unglaublich, dass einem so unwichtig scheinenden Detail solche Aufmerksamkeit zukommt und so viel Erfindungsgeist investiert wurde, um eine Störung künstlich zu erzeugen. Und warum konnte man während 70 Jahren dennoch darauf verzichten? Motion Blur bewegt sich auf dem schmalen Grat zwischen Konvention und natürlichem Wahrnehmungshintergrund und wirft damit eine Reihe von theoretischen Fragen auf, auf die ich in der Schlussbetrachtung näher eingehen werde. Einerseits nimmt auch das menschliche Auge mit seiner Trägheit rasch bewegte Objekte unscharf und halbtransparent wahr: Wenn man einen Kolibri im Flug beobachtet, sind seine Flügel deshalb kaum sichtbar. Dennoch ist dieser Effekt bei der Filmaufzeichnung wesentlich ausgeprägter, da die zeitliche Auflösung des Films mit $40 \mathrm{~ms}$ fast halb so groß wie jene des menschlichen Sehens ausfällt, die bei ca. $20 \mathrm{~ms}$ angesetzt wird. Elektronische Medien, die auf Zeilenabtastung basieren, unterscheiden sich in ihrer Verzeichnungscharakteristik von Bewegungsaufnahmen signifikant vom filmischen Eindruck. 
Simulierter Motion Blur auf digital modellierten Effekten kann wie das gerechnete Korn dem Zweck dienen, die Differenz zwischen analog und digital produzierten Bildteilen zu verwischen und damit einen ,PopOut-Effekt $^{`} \mathrm{zu}$ vermeiden, der den Zuschauer unweigerlich auf die künstliche Herkunft der CGI hinweisen würde. In der Mehrzahl aller Fälle dürfte diese Erklärung zutreffen. Wiederum wird diese vordergründig plausible Zielsetzung aber in einigen Fällen ad absurdum geführt, nicht nur bei den bereits zitierten Dinosauriern, bei denen Steven Spielberg laut Interview den korrekten Motion Blur als unabdingbar für den Realitätseffekt hielt. Unabhängig von der Grundsatzfrage, ob die ,Voluntary Suspension of Disbelief' überhaupt noch greift, wenn $\mathrm{Zu}$ schauer in zahlreichen Hintergrundberichten bis ins Detail über die Techniken der Herstellung informiert werden, lässt sich ein Phänomen ausmachen, das der ursprünglich mimetischen Funktion diametral entgegenzustehen scheint: die Übertreibung.

Übertreibungen des Motion Blur kommen in verschiedenen Abstufungen vor, von relativ diskret am Rande der Konvention angesiedelten Versionen bis zu grotesk ausgestellten Effekten. Verschiedene Tierarten beispielsweise, die dem Protagonisten in THE MuMMY (USA 1999, Stephen Sommers) das Leben schwer machen, vom massiven Aufgebot an Heuschrecken in biblischer Tradition bis zu den Heerscharen von zerstörungswütigen Skarabäen, wischen in ungewohnt unscharfer Manier durchs Bild.

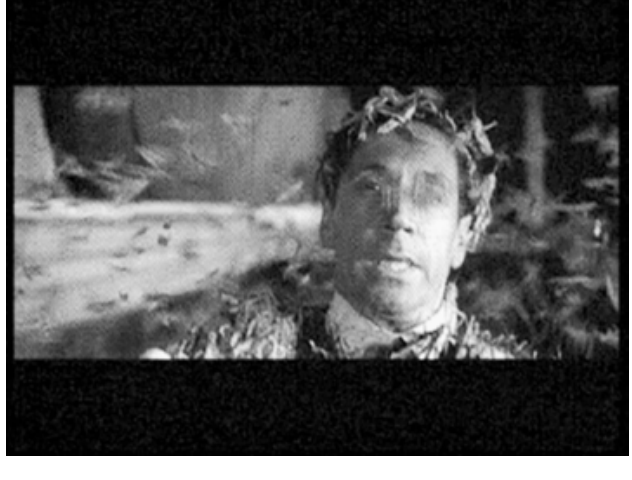

Abb. 1: Still aus THE MUMMY

In der Mehrzahl ergießen sich prozedural animierte Ströme über Stock und Stein oder schwirren ganze Wolken durch die Lüfte. Hier und da aber wird ein einzelnes Exemplar - von den Animatoren Hero-Scarab 
genannt - sehr scharf und wenig bewegt abgebildet, und dann drängt sich die Vermutung auf, dass die 3D-modellierten Skarabäen der genaueren visuellen Analyse nur bedingt standhalten, dass der Motion Blur die harsche Schärfe des computergenerierten Bildes mildern soll. Skarabäen, auch wenn sie nicht in Massen in unserer Alltagswelt vorkommen, sind doch einem wesentlich genaueren Vorstellungsbild unterworfen als Dinosaurier und werden darum vom Zuschauer genauer analysiert als prähistorische Tiere, die bei ihrem bildhaften Erscheinen erst einmal ungläubiges Staunen hervorrufen dürften.

In einigen Fällen geht die Übertreibung noch weiter, wie zum Beispiel dort, wo Neo in THE MATRIX (USA 1999, Larry und Andy Wachowski) Waffen bestellt, die in phantastischer Geschwindigkeit geliefert werden, wobei diese Geschwindigkeit analog zu grafischen Darstellungen in Kunst und Comic mit maximalem Motion Blur verdeutlicht wird.

Ein auffällig wirkender Motion Blur wird aber auch dort appliziert, wo es darum geht, psychische Vorstellungsbilder in Bildmetaphern zu übersetzen. Mit analogen oder teildigitalen Mitteln wird das bereits seit einiger Zeit praktiziert, indem solche Szenen mit einer Bildrate von weniger als zehn Bildern pro Sekunde aufgenommen werden, wodurch sich die Bewegungsunschärfe mehr als verdoppelt. Anschließend kopiert man jedes dieser Bilder mehrfach oder interpoliert dazwischen liegende Frames mit der entsprechenden Software, die es als Motion-EstimationTools auf dem Markt gibt, so dass das Bild in Zeitlupe erscheint. Elektronische Kameras haben eine solche Aufnahmetechnik unter dem Begriff ,Smear-Effekt" teilweise schon vorprogrammiert. Dieses Prinzip simulieren Bilder einer Sexszene in Fight Club (USA 1999, David Fincher), in dem der Protagonist sein abgespaltenes Alter Ego in entfesselter Vereinigung mit Marla Singer imaginiert. „It was supposed to look like one of the heads from Mount Rushmore was fucking the Statue of Liberty." 6 Die grotesk wirkende Umformung des Körpers sollte eine fantastische, traumähnliche Qualität haben. Aufnahmen mit einer Belichtungszeit von $1 / 250 \mathrm{~s}$ zu Beginn und am Ende des Aktes wurden kombiniert mit Fotos, die man während $2 \mathrm{~s}$ belichtete. Die Fotos dienten anschließend als Ausgangsmaterial für eine 3D-Modellierung und -Animation der Szene.

6 David Fincher zitiert nach: Martin, Kevin H.: „A World of Hurt“, in: Cinefex, Jg. 19, Nr. 80 (1999) S. 125. 


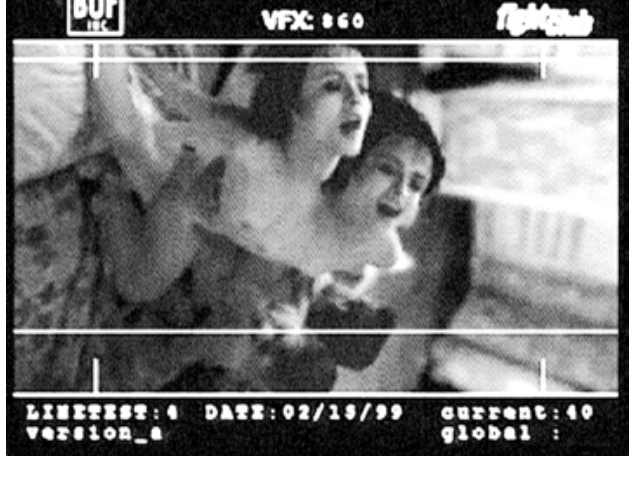

Abb. 2: Still aus FIGHT CLUB

Ein ähnliches Prinzip wird in der übermenschlichen Bewegungsfähigkeit des Agent Smith aus THE MATRIX wirksam, der im Shootout zur vielköpfigen Hydra mutiert. In den so genannten ,Bullet-Time-Szenen“ hingegen, den eingefrorenen Momenten, die inzwischen zum Markenzeichen der Matrixwelt stilisiert und unzählige Male kopiert wurden, wird ein gegenteiliger Effekt wirksam. Das Kamera-Array - eine Serienschaltung von bis zu 120 Kleinbildkameras, die ein Computer in exakt definierten Zeitintervallen auslöst - hat die einzelnen Bilder mit der Belichtungszeit von 1/1.000s aufgenommen, um jede Bewegungsunschärfe zu unterdrücken. Sowohl im Smear-Effekt als auch in den eingefrorenen Momenten entstehen paradoxe Eindrücke entweder durch extreme Verlangsamung kombiniert mit maximalem Motion Blur oder durch extreme Beschleunigung mit minimaler Bewegungsunschärfe. Beide Wirkungen belegen den ungeahnt bedeutsamen, wenn auch völlig verinnerlichten Status einer analogen Störung, die ein durchschnittlicher Zuschauer nicht einmal bewusst wahrnimmt. Trotzdem hat sie sich im Lauf einer hundertjährigen Geschichte analoger Bildaufzeichnung offenbar fest mit dem Medium verbunden.

Ähnliches gilt für den Shuttereffekt, die leicht ruckartige Wiedergabe von Kamera- oder Objektbewegungen. Er entsteht, weil zwischen den einzelnen Bildern Zeitintervalle liegen, die von der Kamera nicht erfasst werden. Heutige digitale Kameras nehmen wie herkömmliche Filmkameras Vollbilder auf, sie arbeiten im so genannten ,progressiven Modus‘. Zudem kann eine Flügelblende zugeschaltet werden, die elektronisch den Shuttereffekt simuliert. Diese Anordnung unterscheidet das HD-24p-Format in seinem Look deutlich von herkömmlichen Videoverfahren, die im so genannten ,Interlaced-Modus‘ je zwei Halbbilder zu 
einem Bild kombinieren. Der progressive Vollbildmodus stellt eine wichtige Voraussetzung für die Herstellung des Film-Looks dar. Theoretisch wäre eine Bildrate von 50 Vollbildern pro Sekunde wünschbar, um Bewegungen möglichst fließend darzustellen. Dieses Desiderat scheitert im Moment jedoch sowohl an der analogen Distributionsform als auch am Wunsch des Publikums, im Kino weiterhin einen gewohnten Bildeindruck vorzufinden.

\subsection{Diffusion, Lens Flares, optische Verzeichnungen, Vignettierungen}

Computergenerierte Bilder wirken frappierend scharf. Nicht nur weil wie bereits erwähnt - statistisch ausgleichende Prozesse des zufällig verteilten Filmkorns wegfallen, sondern auch weil die Pixel das Licht wie Gefäße absorbieren, während die Halogenidkristalle in der analogen Emulsion zu einer leichten, aber essenziellen Lichtdiffusion führen. Dieser Diffusionseffekt wurde im klassischen Hollywoodkino durch weitere Maßnahmen ausgebaut. Eine Reihe von weichzeichnenden Filtern, Fettschichten vor den Objektiven oder eine bestimmte Sorte eines schwarzen Dior-Damenstrumpfs unterstützen den Schmelz klassischer Staraufnahmen, indem sie Unebenheiten ausgleichen und durch Diffusion des Spitzlichts die glänzende Aura des Stars unterstützen. Bewusst oder unbewusst knüpft dieser Abbildungsmodus an die Ikonografie des Heiligenbilds an, besonders an jene romantisch bis kitschigen Darstellungen, in denen sich die Sonnenstrahlen ihren Weg durch eine dräuende Wolkenschicht auf das Haupt des Heiligen bahnen.

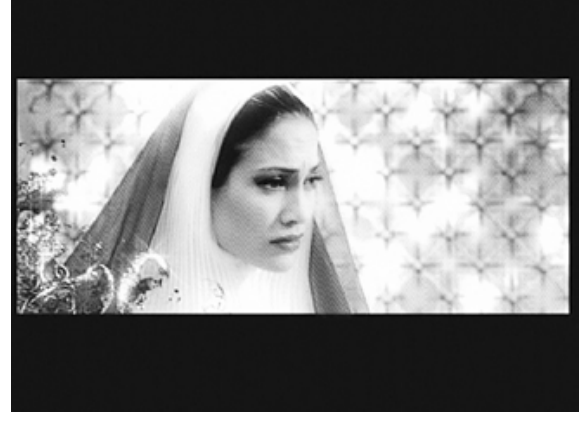

Abb. 3: Still aus THE CELL 
Kein Wunder also, dass man von einem Halo spricht, wenn wie in Avalon (Japan/Polen 2001, Mamoru Oshii) die Schwärzen Höfe bilden oder wie in MINORITY REPORT das Spitzlicht diffus ausbrennt.

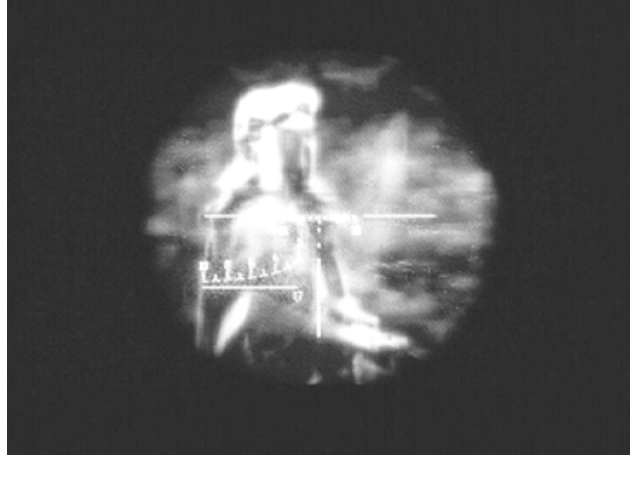

Abb. 4: Still aus AVALON

In den romantischen Darstellungsmodus wurde später eine Störung des technischen Apparats integriert, die man klassischerweise vermieden hat: so genannte ,Lens Flares' oder Objektivreflexe. Sie entstehen, weil Objektive aus einer Reihe von Linsen bestehen, in denen sich direkt einfallende Lichtstrahlen mehrfach brechen und reflektiert werden. Dabei bilden sich unregelmäßige, häufig sechseckige Lichtflecken, die sich bei Kamerabewegungen in komplexen Mustern über das Bild verschieben. Da es sich bei der auslösenden Lichtquelle meist um Sonnenlicht handelt, werden Lens Flares mit angenehm warmen Temperaturen und südlich angehauchten Idyllen assoziiert.

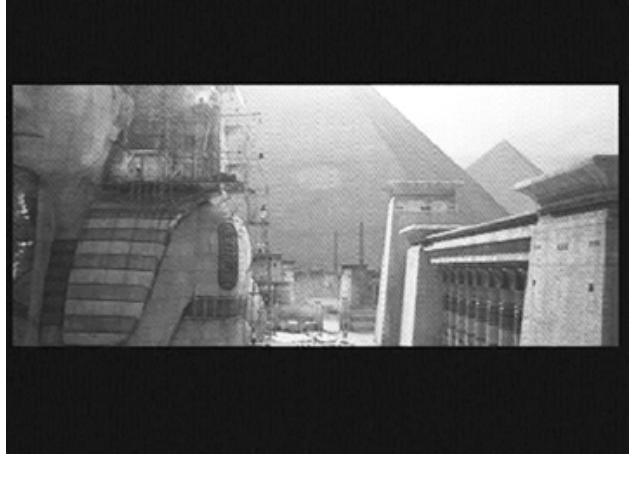

Abb. 5: Still aus THE MUMMY 
Eine weitere Quelle von Lens Flares können Autoscheinwerfer in der Dunkelheit oder Sterne in den Galaxien des Universums sein, die je eigene Assoziationen befördern. In volldigitalen Bildern knüpfen Lens Flares nicht nur an diese Assoziationen an, sondern sie suggerieren gleichzeitig eine materiell vorhandene Kamera, mithin die Präsenz eines Beobachters im Bild, da sich durch den Lens Flare die dreidimensionale Materialität der Kamera ins Bild einschreibt. In Bildern, die im Compositingverfahren montiert werden, wirken Lens Flares als ,Suturing Devices', als verbindende Elemente, welche die Bildteile durch das Prinzip des ,gemeinsamen Schicksals', das aus der Gestaltpsychologie bekannt ist, zu einem einheitlichen Wahrnehmungsgegenstand verschmelzen. Inzwischen ist der Gebrauch von Lens Flares besonders in modellierten Einstellungen so verbreitet, dass der Effekt fast schon als Hinweis auf die künstliche Entstehung des Bildes zu lesen ist - eine Wirkung, die verstärkt wird, indem diese Reflexe auffällig wohlgeordnet im Bild auftauchen.

Optische Verzeichnungen wie die Krümmung von Linien gegen den Bildrand hin, die bei Weitwinkelaufnahmen auftreten, gehören zu jenen analogen Störungen, die noch weniger Beachtung finden als der Shuttereffekt oder die Bewegungsunschärfe. Dennoch haben sie sich untrennbar mit unserer Wahrnehmung von analogen Bildaufnahmen verknüpft und sind besonders dort spürbar, wo Kamerabewegungen stattfinden. Perspektivische Verkürzung - ein Effekt, der durch Teleobjektive hervorgerufen wird - lässt sich ebenfalls der Kategorie von optischen Verzeichnungen zuordnen. Offenbar sind diese Verzeichnungen von geringer Wichtigkeit und werden höchstens dann auf das digitale Bild gerechnet, wenn es um die Anpassung an ein analoges Umfeld geht - mit einer bedeutsamen Ausnahme: THE MATRIX. Optische Verzerrungen sind dort nicht nur verschämte Störungen am Rande, sondern werden geradezu zum Bildinhalt. Zahlreiche Spiegelungen in gekrümmten Flächen, in Spiegeln, Löffeln, Sonnenbrillen reizen die tonnenförmigen Verzerrungen bis zum Exzess aus, und als Krönung erscheint ein Stadtbild in Vogelperspektive, indem die Wolkenkratzer als Fischaugenaufnahme in ein kreisrundes Bildfenster gepfercht werden.

Spiegelungen, die in diesem Film leitthematisch die verwirrende Verstrickung von Simulation und Wirklichkeit symbolisieren, entstehen bemerkenswerter Weise nicht auf glatten, sauberen Oberflächen, sondern verzerrt und schmutzig auf Alltagsgegenständen, und trotzdem sind alle diese Spiegelungen digitale Kompositionen, welche die Verzerrungen nur simulieren, statt sie abzubilden. So nämlich ließen sie sich nach Be- 
lieben extrem entstellen und nehmen damit wiederum ein anderes Motiv dieses Films auf, die Deformation des Körpers.

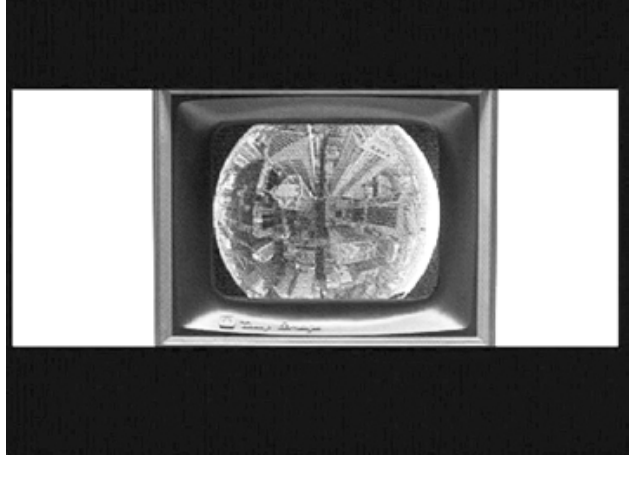

Abb. 6: Still aus THE MATRIX

Als Vignettierungen schließlich bezeichnet man die Abnahme von Licht zum Bildrand und besonders zu den Bildecken hin, der besonders bei Weitwinkelaufnahmen zu beobachten ist. Diese letzte durch Objektive bedingte Störung in meinem Streifzug durch die Phänomene kommt heute in der professionellen Praxis bei analogen Bildaufnahmen kaum mehr vor. Entsprechend wirken sie als Hinweise entweder wie in MOULIN ROUGE auf eine historische oder aber auf eine amateurhafte Aufnahme.

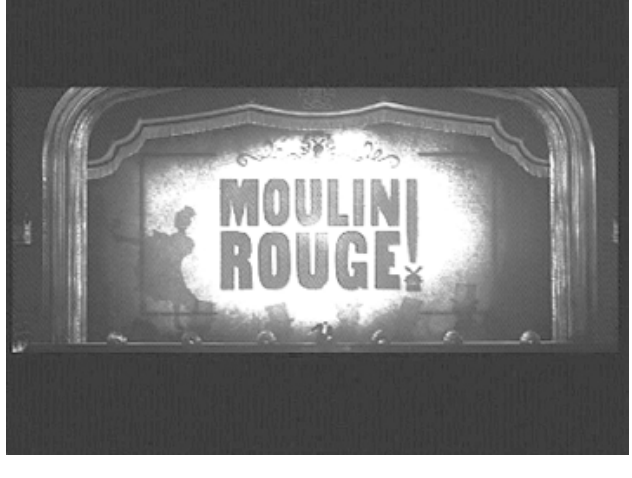

Abb. 7: Still aus MOULIN ROUGE 
Dem Kontext der historischen oder amateurhaften Aufnahme zuzurechnen sind Erinnerungsbilder, die den Fotos vertrauter Menschen und Situationen nachempfunden und darum mit Vignettierungen versehen sind. Das heißt, die Erinnerung oder vielmehr die Darstellung von Erinnerung knüpft an die formale Erscheinungsweise eines medial repräsentierten Bildergedächtnisses an. Speziell komplex ist diese Kodierung in MinORITY REPORT, in dem drei Wahrsager, so genannte Precogs, die Zukunft nach Verbrechen absuchen, damit das Precrime-Department diese Verbrechen verhindern und die mutmaßlichen Täter noch vor der Tat verhaften kann. Diese Zukunftsbilder lehnen sich an Erinnerungsbilder an. Sie gründen auf psychologischen Konzepten von Traum und Erinnerung. Die Vignettierung symbolisiert nicht nur einen Mangel in der Darstellung, sondern zugleich Konzentration auf den wesentlichen Bildinhalt, der sich - aus einem dunklen Umfeld herausgelöst - ins Zentrum der Wahrnehmung schiebt und ein Spiel zwischen Verdrängung und Fokussierung nachzeichnet.

\subsection{Rauschen, Zusammenbruch des Signals}

In einer Szene aus WAG THE DOG (USA 1997, Barry Levinson) ist eine groteske Anwendung von Visual Effects zu sehen. Eine junge Schauspielerin mimt vor einer Bluescreen ein albanisches Flüchtlingsmädchen, das sich mit einem Kätzchen im Arm vor den Aggressoren rettet. Die Szene - als dokumentarische Aufnahme für die Nachrichten geplant soll in der Bevölkerung Emotionen wecken und eine militärische Intervention der USA rechtfertigen, mit welcher der Präsident von einer Sexaffäre ablenken will. Vor den Augen des Zuschauers entsteht eine fiktive Dokumentation über einen Krieg, den es gar nicht gibt. Aus Datenbanken werden Kriegsschauplätze heruntergeladen und auf persönlichen Wunsch des Präsidenten eine weiße Katze eingefügt, das alles in wundersamer Verkürzung des effektiven Arbeitsaufwands und mit beispielloser Leichtigkeit. Eingebettet in eine klassische Nachrichtensituation wird der Bericht vom Fernsehen gesendet, nun zusätzlich mit Rauschen und einem kurzfristigen Drop-Out des Signals versehen. Solche elektronischen Bildstörungen, vor allem Rauschen und Signalunterbrechung sind längst zum Zeichen des Authentischen mutiert, besonders dort, wo dieses Authentische erzählt, also konstruiert werden soll. Insofern ist die beschriebene Szene aus WAG THE DOG ein seltenes Lehrstück, in der die Zeichenhaftigkeit der elektronischen Störung zum Gegenstand der Erzählung selbst erhoben wird. Dabei sind solche Eingriffe Legion. Wo immer 
Bilder medial gestaffelt über einen Bildschirm im Bild zu sehen sind, werden sie mit medientypischen Störungen angereichert. Damit suggerieren sie stressige Aufnahmebedingungen, wie sie in der Livesituation in Krisengebieten vorkommen, wo die Reporter nur unter schwierigsten Bedingungen drehen können, ständig bedroht durch Stromunterbrechungen und Übertragungsfehler.

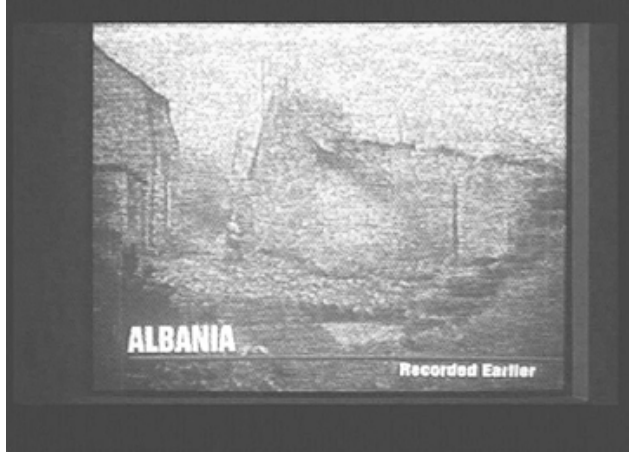

Abb. 8: Still aus WAG THE DOG

Das war allerdings nicht immer so. Eines der frühesten Beispiele einer medialen Staffelung dürfte das Bildtelefongespräch aus METROPOLIS (Deutschland 1927, Fritz Lang) sein. In MeTROPOLIS jedoch trug diese technische Errungenschaft noch die Züge des futuristischen Traums und sollte die Zuschauer durch Staunen belehren. Längst ist in der filmischen Darstellung auch die Zukunft nicht mehr, was sie einmal war und so ist es zu erklären, dass beispielsweise in MINORITY REPORT das derzeit noch inexistente Technikgadget der interaktiven 3D-Projektion mit einem Zeilenmuster in geringer Auflösung versehen wird. „Steven wanted to see mistakes. [...] He wanted to see the workings of these things. “" ${ }^{77}$ Und auch in THE MATRIX entsprechen die Bildschirme der Zukunft in Auflösung und Transparenz nicht den Prophezeiungen der New Economy. Vielmehr könnte man diese mit Störungen überfrachteten Darstellungsweisen als Ausdruck eines massiven Technikskeptizismus werten, wie überhaupt die zukünftigen Welten im Science-Fiction-Film seit BLADE RUNNER ihre polierten Oberflächen mehrheitlich abgelegt haben. Davor hatte schon George Lucas den Begriff von der used future geprägt. Mit einem rostigen und staubigen Look wollte er sich bewusst

7 Scott Farrar zitiert nach: Cinefex, Jg. 22, Nr. 91 (2002) S. 48. 
von der Science-Fiction-Ästhetik früherer Zeiten distanzieren, wenn es auch völlig verfehlt wäre, dem STAR WARS-Universum eine technik- und zivilisationskritische Haltung anzudichten. Oder um es anders zu formulieren: Nicht überall decken sich Form und Inhalt, nicht überall sind sichtbares Rauschen und Drop-Outs Ausdruck einer pessimistischen Haltung bezüglich der langfristigen Auswirkungen von technologischen Entwicklungen auf die Befindlichkeit von Individuum und Gesellschaft. Vielmehr ist der Retro-Look oftmals nicht mehr als ein modischer Trend, der in der Konsumwelt seit Jahren seine Nischen hat, von den nachgebauten Antiquitäten bis hin zur ,stone-washed' Jeans.

\section{Ein Panoptikum möglicher Interpretationen}

Nun lassen sich die Phänomene unter verschiedenen Gesichtspunkten betrachten, nach einem Panoptikum der Phänomene folgt also ein Panoptikum möglicher Betrachtungsweisen, von abbildungstheoretischen Fragestellungen oder filmhistorischen Überlegungen über wahrnehmungspsychologische Aspekte bis hin zur ideologischen Komponente des bedeutungserzeugenden Apparats. Weil meine Abhandlung ein ,Work in Progress' ist und lediglich einen Ausschnitt aus einem laufenden Forschungsprojekt problematisiert, werden möglicherweise mehr Fragen als Antworten auftauchen.

Fangen wir mit der Abbildungstheorie an, die im Zusammenhang mit dem ,digitalen Bild" seit Mitchells Reconfigured Eye besonders den historisch gewachsenen Status des fotografischen Bildes als Spur einer vergangenen Wirklichkeit diskutiert, mithin also den Aspekt der Wahrheit eines Bildes mit seinen ethischen und politischen Implikationen. Diese Problemzone ist einerseits gewaltig und gleichzeitig im Rahmen der Fiktion von untergeordneter Bedeutung. Denn das fiktionale Filmbild hat schon immer gelogen, schon immer bediente es sich verschiedener Techniken der Verfälschung vor, in und nach der Kamera. Die einzige Frage, die sich stellte, war nur, wie kohärent und plausibel die Lüge erschien und welche Strategien das Kino entwickelte, um den Zuschauer zu umgarnen und noch in die ungewöhnlichsten Fantastereien zu verspinnen. In den klassischen Theorien, die sich mit den Transformationen des filmischen Bildes beschäftigten, schwang immer das Paradigma mit, dass der kinematografische Apparat alles daran setze, seine eigene Existenz zu verbergen. Es schien also, als ob größtmögliche Transparenz des Apparats das unausgesprochene Ziel aller technischen Entwicklungen bilden 
sollte, ein Ziel, dem die Hochglanz-Breitwand-Produktionen der 50er und 60er Jahre des 20. Jahrhunderts so nah wie nie waren.

Wie ist es zu erklären, dass längst überwundene Störungen, die den Apparat als solchen sichtbar machen, plötzlich eine Renaissance erleben? Heißt das, dass die klassischen Thesen falsch sind, oder sind sie lediglich überwunden und wenn ja warum?

Wenn man die Grundpfeiler Kausalität und Ähnlichkeit betrachtet, die klassischerweise eine gelungene Abbildung bestimmen, so ist die imitierte analoge Störung in beiden Fällen etwas Aufgepfropftes, dem tatsächlichen Prozess Überlagertes. Sie weist nicht auf den Abbildungsgegenstand hin, sondern auf ein Abbildungsverfahren und bildet damit eine mehrfache Referenzialität, man könnte auch sagen eine Referenzialität höherer Ordnung, die jenem Komplex zuzuordnen ist, den Barthes als ,Mythos ‘* bezeichnet hat. Mythen treten in dieser Konzeption immer dann auf, wenn eine kulturelle Praxis zur reinen Materie hinzutritt und ihre Bedeutung verändert. Diese kulturelle Praxis figuriert auch unter dem Begriff ,Konvention“.

Damit eröffnen sich meiner Argumentation zwei neue Bedeutungsfelder: Die Kategorie des Natürlichen vs. die Kategorie der Konvention. In Fragen umformuliert: Ist das analoge fotografische Bild ,natürlicher oder ist es lediglich stärker konventionalisiert als das digitale? Und wo liegen überhaupt die Unterschiede? Beide Kategorien beschreiben Formen der Transparenz, man könnte auch sagen Formen der Selbstverständlichkeit, mit denen Rezipienten ein ästhetisches Angebot annehmen und verstehen. Das Natürliche wäre dabei jene Transparenz, die durch die Alltagserfahrung im direkten Umgang mit der Umwelt gegeben ist, während das Konventionelle seine Transparenz durch Gewöhnung an eine regelhafte Transformation erlangt. Die analoge Störung im digitalen Bild kann in beiden Zusammenhängen gesehen werden. Wieder und wieder diskutieren Visual Effects Artists die Abweichung von der allzu evidenten, allzu regelhaften Ordnung als Kategorie des Natürlichen. Es gebe, so argumentieren sie, in der Natur keine geraden Linien, und die Natur verfüge über eine unendliche Komplexität. Wo immer CGI entsteht, wird Chaos dazugerechnet, werden gleichmäßige Linien und Flächen Alterungs- und Witterungsprozessen unterworfen. Störungen, die analogen Abbildungsverfahren nachgebildet werden, helfen mit, diese evidente Regelhaftigkeit zu durchbrechen, gerade Linien und saubere Flächen in das milde Licht eines gewissen Chaos einzutauchen. Gleich-

* Anm. d. Hrsg.: Vgl. Barthes, Roland: Mythen des Alltags [1957], Frankfurt a. M. 1964, S. 85-151. 
zeitig sind diese Störungen auch ein Element des historischen Konventionalisierungsprozesses, besonders dort, wo sie in jenem kulturellen Rahmen erscheinen, der sie hervorgebracht und geprägt hat, in unserem Fall das Kino, genauer: die fotorealistische Fiktion im Kino, denn der Animationsfilm hat seine eigenen Konventionen entwickelt, die sich speziell hinsichtlich meiner Fragestellung unterscheiden.

Mit der Konventionalisierungsfrage betrete ich jedoch schon das Feld der Filmhistorie. In einem Korpus von derzeit rund hundert hybriden, analog-digitalen Filmen fallen bis dato die Mehrzahl jener Werke, die das digitale Bild $\mathrm{ab}$ und $\mathrm{zu}$ in seiner ureigenen Qualität feiern, ins Genre des Science-Fiction-Films. Dazu gehört besonders der Film TrON (USA 1982, Steven Lisberger), bei dem sich die umgekehrte Strategie beobachten lässt: eine Reihe von ,digitalen“ Bildern wurden analog erzeugt und von Hand in zeitraubenden Verfahren nachgebildet. TRON war nicht nur ein Flop, man könnte sogar von einem ,Tron-Schock` sprechen. Pioniere digital erzeugter Bildsequenzen wie James Cameron oder Steven Spielberg haben einige Jahre nach TRON in ihren Filmen peinlich darauf geachtet, dass diese Bilder eine organische Anmutung hatten und sich quasi unsichtbar in den weiteren ästhetischen Kontext des Films einfügten. Zwar soll das digitale Bild im filmhistorischen Kontext als virtuose Errungenschaft ausgestellt werden, gleichzeitig soll sich in dieser Virtuosität kein Bruch mit der analogen Tradition manifestieren. Regelmäßig fallen im DVD-Bonusmaterial zu digitalen Visual Effects Attribute wie ,magic', ,incredible' oder ,groundbreaking', welche die revolutionäre Kraft der digitalen Errungenschaften geradezu auf einen Sockel stellen. Gleichzeitig minimieren dieselben Praktiker die Differenzqualität des digitalen Bildes, indem sie es analogen Störungen unterwerfen.

Seit jeher wird der Opposition sichtbar/unsichtbar aber auch eine ideologische Komponente zugeschrieben. Während die sichtbare Transformation unter dem Stichwort der Verfremdung einen ideologisch einwandfreien Bewusstseinsprozess im Zuschauer auslöst, ihn aus der tumbpassiven Konsumption befreien und der bewussten Partizipation am Kunstwerk zuführen sollte, gilt die Maskierung des bedeutungserzeugenden Apparats als ideologisch bedenklich. Nun dienen Störungen aber beiden Zielsetzungen. Teilweise sollen sie digitales Material nahtlos in ein analoges Umfeld integrieren, wie zum Beispiel in FORREST GUMP (USA 1994, Robert Zemeckis), teilweise führen sie aber zu einer sichtbaren Transformation eines ursprünglich neutralen Darstellungsmodus, wie die grotesken Motion-Blur-Effekte in FIGHT CLUB. Nicht selten äußern sich Regisseure explizit dahingehend, dass sie allzu glatte, 
unauffällige Kompositionen aufrauen wollen. So wies James Cameron die Visual-Effects-Spezialisten an, einen allzu wundersamen Heilungsprozess des T-1000-Cyborgs in TERMINATOR 2 - JUDGMENT DAY zu verschmutzen: „Don't think of yourselves as Industrial Light and Magic, think of yourselves as Industrial, Light and Dirt." ${ }^{8}$ Im Rahmen der Sichtbarkeits-/Unsichtbarkeitsdebatte geht es bei solchen Aufrauungsstrategien jedoch meist darum, das Material aus seiner digitalen Qualität in analoge Gefilde zu verschieben. Anders ausgedrückt sollte die Sichtbarkeit nicht auf das tatsächliche Medium, sondern auf ein simuliertes analoges hinweisen, dem eine organische Ästhetik zugeschrieben wird. Damit schließt sich der Kreis zu den Überlegungen, die ich hinsichtlich der Konventionalisierungsfrage diskutiert habe. Es ließe sich also feststellen, dass gegenwärtige Störungsverfahren, selbst wenn sie so weit getrieben werden, dass sie den Rahmen des Unsichtbaren verlassen, in letzter Instanz doch dem Zweck dienen, mathematisch automatisierte Abläufe mit einem unregelmäßigen, teilweise sogar individuell von Hand erzeugten Raster zu überlagern.

Dieser Wunsch hat seine Wurzeln vielleicht nicht nur in den filmhistorisch begründeten Konventionen, sondern ebenso sehr in der Beschaffenheit des menschlichen Wahrnehmungssystems. Dieses Wahrnehmungs- und Informationsverarbeitungssystem ist nämlich evolutionär bedingt auf Veränderungen programmiert. Bleiben Reize stabil oder gleichförmig, werden sie ausgeblendet, während Veränderungen Aufmerksamkeitsreaktionen auslösen. Einige Eigenschaften des traditionellen Filmmaterials wie der leicht instabile Bildstand, aber auch die Zufallsverteilung des Filmkorns scheinen perfekt an diese Anforderung angepasst zu sein. Sie füttern das Wahrnehmungssystem auf der Mikroebene beständig mit leichten Schwankungen und zwingen es so zu fortgesetzter Aufmerksamkeit. Nun ist diese Argumentation aber gewagt und vielleicht auch nicht haltbar. Immerhin gibt es viele digitale Darstellungsformen, die funktionieren, obwohl sie auf solche Mikroschwankungen verzichten, zumindest alle jene, die nicht nur vom Computer erzeugt, sondern auch am Computer konsumiert werden, wie beispielsweise Spiele oder Programmoberflächen. Ohne zu sehr in die Materie des Computerspiels eindringen zu wollen, konstatiere ich jedoch auch dort immer mal wieder einen Rückgriff auf analoge Störungen, gerechnetes Korn, Lens Flares, Diffusion oder Vignettierungen, wie beispielsweise im MYST RIVEN-Universum, das insgesamt mit den Konventionen des bereits beschriebenen Retro-Looks spielt.

8 James Cameron zitiert nach: Cinefex, Jg. 11, Nr. 47 (1991) S. 33. 
Mögen die Phänomene im Einzelnen noch so unterschiedlich sein, so sind sie doch mehrheitlich funktionalisiert oder sogar zeichenhaft zugespitzt und damit konventionalisiert. Dennoch sind Mainstreamfilme längst nicht mehr notwendigerweise geschlossene Werke, in denen sämtliche Bilder und Töne einem zielgerichteten Erzählgestus unterworfen sind. Längst haben sie Brüche aus der avantgardistischen Tradition integriert, arbeiten sie mit Fragmentierungen, Ambivalenzen, Verfremdungen, Überraschungen, um den Zuschauer zu fesseln und dessen Emotionen zu wecken. In dieser Entwicklung wird ein Muster sichtbar, das vielen ästhetischen Entwicklungen gemein ist. Wenn nämlich Grundprinzipien eines Darstellungscodes gemeistert sind, wenn also beispielsweise Spielfilme dank eines hochkomplexen Regelwerks mit einem hohen Maß an fließender Eleganz überzeugen, müssen technische oder ästhetische Innovationen einer allzu drastischen Konventionalisierung entgegenwirken, um den mediengewohnten Zuschauer weiter zum Konsum zu verführen.

Es stellt sich jedoch die Frage, ob die Störung des digitalen Bildes in diesem Licht gesehen werden darf. Ist das digitale Bild tatsächlich schon so stark konventionalisiert, dass wir es einer Revision unterwerfen müssen? Haben wir es, anders gefragt, kulturell bereits gemeistert? Oder sollen die analogen Störungen die digitalen Ecken und Kanten weichspülen? Handelt es sich, anders gefragt, um einen postmodernen Romantizismus, um Nostalgie und Verklärung? Oder geht es am Ende sogar um jene subjektiven Transformationen eines neutralen Materials, die spätestens seit dem ausgehenden 19. Jahrhundert unter dem Begriff ,Kunst ${ }^{`}$ als Ausdruck einer eigenwilligen kreativen Instanz gedeutet werden? 